\title{
Nasopharyngeal Swab Specimen
}

National Cancer Institute

\section{Source}

National Cancer Institute. Nasopharyngeal Swab Specimen. NCI Thesaurus. Code

C155831.

A biospecimen collected from the back of the throat by swabbing. 